\title{
Online State Estimator for Three Phase Active Distribution Networks Displayed on Geographic Information System
}

\author{
Dimas Fajar U. P., Indri Suryawati, Ontoseno Penangsang, Adi Suprijanto, and Mat Syai'in
}

\begin{abstract}
To improve the operating performance of a distribution network, on line monitoring is required. For this purpose, sensors (metering devices) are installed. To reduce the number of sensors, state estimation approach can be used to estimate the voltage of buses which do not have sensors. This paper proposes online state estimator for three phase active distribution networks using Neural Network and displayed the results on Geographic Information System (GIS). Neural Network based state estimation is used to estimate the bus voltages by using learning approach from power flow patterns. K-matrix three phase distribution power flow is used in this method as an analytical tool. The K-matrix approach is combined with Particle Swarm Optimization (PSO) in handling a Distributed Generation (DG) which is operated as a voltage controlled (PV) bus. The test results show that the proposed method can reduce the number of sensors significantly (almost $50 \%)$.
\end{abstract}

Index Terms-State estimator, neural network, K-matrix, PSO and GIS.

\section{INTRODUCTION}

Electrical distribution systems is a part of power systems that directly connected to the consumers. In some electrical distribution systems, can consist of a huge number of bus. Due to this reason, monitoring and controlling the electrical distribution system for real time becoming very important to improve its operating performance.

To build this automation systems, huge number of sensor are needed to monitor all part in the systems. But if the sensor installed at all buses, investment cost become huge. To reduce the investment cost, the numbers of installed sensor in the bus need to be reduced. To reduce the number of sensors, state estimation approach can be used to estimate the voltage of buses which do not have sensors.

The concept of state estimator was first discovered by Fred Schweppe on the transmission system. The basic idea of the concept is to combine the advantages of measurements using an equations system as to find the conditions that may occur on the network, resulting the minimization of the error of sensor and the delay of sensor readings. Vector estimation in transmission systems consists of voltage magnitude and phase

Manuscript received November 20, 2013; revised December 26, 2013 This work was supported by Indonesian Government under Grant Number 013674.155/IT2.7/ PN.08.01/2013.

Dimas Fajar U. P., Indri Suryawati, Ontoseno Penangsang, and Adi Soeprijanto are with the Electrical Engineering Department Institut Teknologi Sepuluh Nopember, Indonesia (e-mail: dimasfup@ee.its.ac.id, indrisuryawati@gmail.com, zenno_379@yahoo.com, adisup@ee.its.ac.id).

Matt Syai'in is with the Department of Marine Electrical Engineering Surabaya Shipbuilding State Polytechnic Surabaya, Indonesia (matt.syaiin@gmail.com). angle [1]-[3]. In [4]-[6], they were developed state estimation for single phase distribution systems, this concept suitable for distribution areas where have balance load system.

Distribution networks have characteristics such as the radial network topology, the high value of $\mathrm{R} / \mathrm{X}$. There is a lateral system (two-phase and single-phase), and usually have unbalanced load system. Newton Raphson power flow and fast decouple which is used to analyze the transmission system can't be applied to the distribution system, these methods are built on the assumption of balanced three-phase system.

Several methods have been developed to analyze the distribution system like FB, loop frame, FFRPF, direct-ZBR, these methods have accurate analysis but can't accommodate PV bus. The integration of renewable energy sources into the distribution system requires an active distribution power flow to analyze a system performance. The three-phase power flow sequence component based method (SPF-NR) easily accommodates PV bus problems. But SPF-NR cannot accommodate lateral system (two-phase network and single phase). K-matrix distribution power flow is a combination of network topology based methods [7] and direct-ZBR method [8]-[12]. K-matrix distribution power flow algorithm is simpler thus more accommodating the changes of the network structure instead of the previous method. In [10] lambda was used to estimate the voltage at some point which is based on the results of measurements of the voltage sensor at other point measurements.

Voltage estimation in [11] is continuation of research on the integration of renewable energy sources (DG) in distribution networks. The operation of the DG as a PV bus, voltage magnitude is maintained at a certain scale. To get the amount of Volt Var, and optimization is done on the PV bus. Meanwhile, to estimate both the voltage magnitude and voltage angle is maintained accordingly measurement results. Then the optimization is done on the value of Watt and Var on the buses to be estimated voltage. Thus no need sensors at each bus to know the voltage of all buses in the distribution system. The method is robust, but slowness in computational.

This paper proposes neural network distribution state estimation for online monitoring integrated by geographic information system (GIS). State estimation is solved using learning approach from K-matrix-PSO load flow patterns. Neural Network (NN) is an intelligent computing algorithm that inspired the workings of nerve cells. All incoming input signal is multiplied by the weighting for each input, then summed and added to the bias. The sum of the incoming activation function of the neuron produces output. NN will be trained to power flow results of K-matrix-PSO, as many as 50 load pattern. The goal is not only improve the accuracy but 
real time measuring. The advantage of GIS for online monitoring; it has two dimension visualization, detailed information of location and flexibility analysis.

\section{Methodology}

\section{A. K-Matrik Power Flow}

Submit your manuscript electronically for review. For ease of illustration, the simple three phase radial distribution system is shown in Fig. 1. There are five bus and bus no 3 as PV bus. but for this step PV bus is ignored. In other hand the network is passive. The system can be easily analyzed using the $\mathrm{K}$ - matrix power flow method.

$\mathrm{K}$ - matrix is a square matrix with size nbranch $\mathrm{x}$ (nbus 1). nbranch is the number of branches and nbus is the number of bus. The principle of K-matrix, are looking for the route from bus to reference (bus 1). K-matrix would be worth-C if the branch is located on the opposite lane with reference, $\mathrm{C}$ is a diagonal matrix $(3 \times 3)$ with diagonal elements are 1 in accordance with the number of phase. $K$-matrix formed Fig. 1 is expressed in equation (1) as follows:

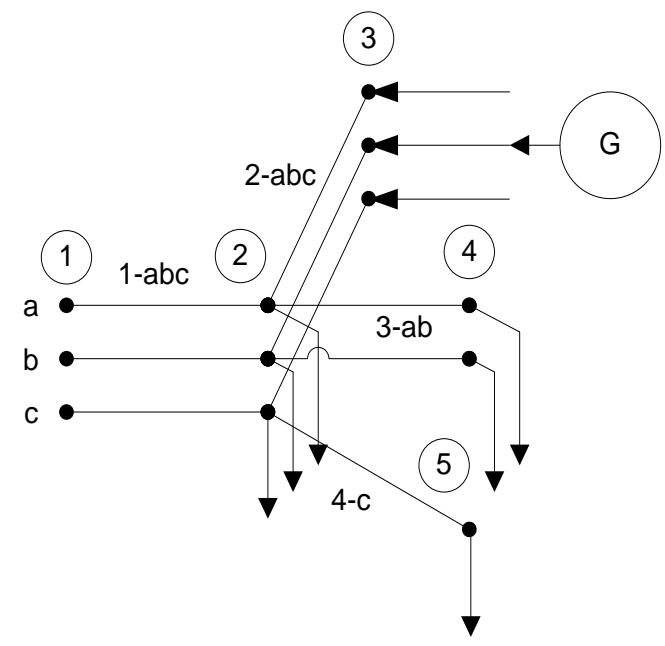

Fig. 1. A simple three phase radial distribution system.

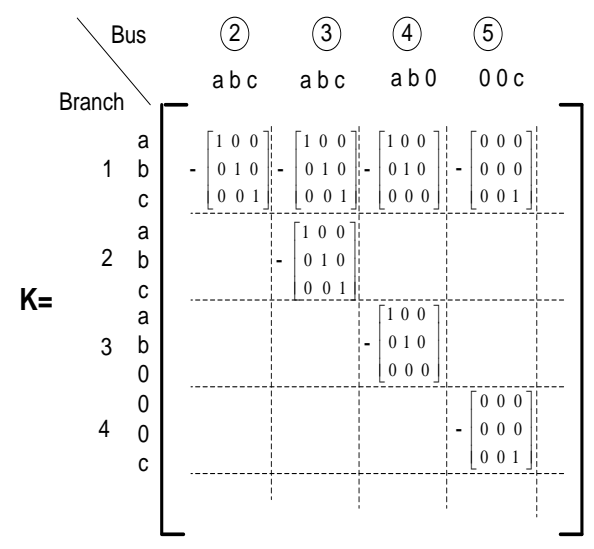

K-matrix power flow algorithm:

1. Input load and network data

2. Build K-matrix

3. Build BCBV matrix, BCBV is negative transpose K-matrix multiply with full branch matrix [].

4. Build DLF matrix, DLF is BCBV multiply with (K-matrix)
5. Then inflows of at each bus was calculated by equation (2)

$$
I_{\text {bus }(l)}^{(k)}=\left(\frac{P_{(l)}^{s h}+j Q_{(l)}^{s h}}{V_{\text {bus }(l)}^{(k)}}\right)
$$

Equation (3) is updated every iteration. Along with the update iteration, it is the result of multiplying the voltage difference between DLF and $I_{b u s}$

$$
\begin{gathered}
\Delta V^{k}=D L F x I_{b u s}^{k} \\
V^{k+1}=V_{\text {bus noload }}-\Delta V^{k}
\end{gathered}
$$

$V_{\text {bus_noload }}$ is the voltage at each bus in the initial conditions, it is set equal to the reference voltage.

6. Check if $\Delta V_{B u s}^{(k+1)}$ are less than a preset tolerance then iteration stop. If it more than a preset tolerance back to point 5.

\section{B. K-Matrik-PSO Power Flow for Active Distribution}

When you submit your final version, after your paper has been accepted, prepare it in two-column format, including figures and tables. K-matrix distribution power flow must be combined with optimization algorithm to estimate the Var value on PV bus. PSO was chosen in this research considering on literature [9]-[21] PSO is robust and simple optimization algorithm

Particle Swarm Optimization (PSO) is heuristic algorithm that inspired by swarm of birds looking for food source [22]. PSO in K-matrix power flow is used for tuning Var in PV bus and keep the voltage constant.

K-matrix-PSO power flow algorithm:

1. Input load, line and generation data

2. Input PSO parameter

3. Random initialization of $\operatorname{Var}(\mathrm{Q})$ for each PV bus.

4. K-matrix Power Flow

5. Evaluate the fitness

6. Getting the best particle (Pbest) and best global particle (Pgbest).

7. Update velocity and Position by equation:

$$
\begin{gathered}
V_{i}^{k+1}=\omega V_{i}^{k}+c_{1} r_{1} x\left(\text { Pbest }_{i}^{k}-X k_{i}^{k}\right)+ \\
c_{2} r_{2} x\left(\text { Gbest }_{i}^{k}-X_{i}^{k}\right) \\
X_{i}^{k+1}=V_{i}^{k}+V_{i}^{k+1}
\end{gathered}
$$

$V_{i}^{k}=$ velocity particle $i$ at $k$ iteration

$\omega=$ weight parameter

$c_{1}, c_{2},=$ acceleration coefficient

$r_{1}, r_{2}=$ random value between 0 until 1

$r_{1} X_{i}^{k}=$ particle position at $k$ iteration

Pbest $_{i}^{k}=$ Pbestparticle $i$ atiterasi $k$

Gbest $_{i}^{k}=$ Gbest particle $i$ iterasi $k$

8. Check constrains Qmin and Qmax. 
9. Check stopping criteria, there are two stopping criteria; maximum iteration and tolerance.

\section{Neural Network Distribution State Estimator}

Neural Network Distribution State Estimator (NNDSE) is designed and tested for real distribution system. It used one of feeder in Surabaya Indonesia. There are ten bus, six bus is load bus and four bus is no load bus. Sensor was placed on two or more bus.

K-matrix power flow patterns divided training and testing, $70 \%$ data as training and $30 \%$ data as testing. In all data use 50 power low patterns. One hidden layer ten node Neural Network back propagation was design.

Artificial neurons are a processing element that functions like neurons in a neural network structure. A number of the input signal is multiplied by each corresponding weights. Then do the sum of all activation function to get the output signal. Suppose there is an input signal and the weights, the output function of the neuron is according the following equation.

$$
F_{\left(x_{1} w\right)}=f\left(w_{1} x_{1}+\ldots+w_{n} x_{n}\right)
$$

Set of neurons made into a network that will serve as a computational tool. The amount of weight between each neuron is connected to be determined the network trained using a set of sample data.

Complete research step is described in Fig. 2. Step 1 until 4 is part of stage A and B. magnitude and angle voltage from 50 load flow pattern from each stage as input and output training testing neural network (step 5). This network was used distribution state estimation (step 6). Module NDSE will export estimation data to database and integrated with GIS to show detail information of location and utility.

\section{TEST AND DISCUSSIONS}

First step result is validation power flow design with commercial software, ETAP. The average different values for all design are 0.001 . That's mean that all design of power flow are feasible.

Neural network distribution state estimation was tested for following test cases.

\section{A. Single Phase Distribution Network}

There are ten bus in feeder of kaliasin (Fig. 3). bus no 3, 5, 6, 8 and 10 are load bus (see Table I).

Case 1 voltage estimation for bus no 5 and 8 consider three input sensor from bus no 3, 6 and 10 .

Case 2 voltage estimation for bus no 5, 6 and 10, sensor was placed on bus no 3 and no 8 .

\section{B. Passive Three Phase Distribution Network}

Case B is state estimation for three phase distribution unbalance network. The data was used is feeder of Kaliasin Surabaya Indonesia. Same with Case A but it consist of three phase unbalance load (see Table II).

Case 1 voltage estimation for bus no 5 and consider three input sensor from bus no 3, 6 and 10 .

Case 2 voltage estimation for bus no 5, 6 and 10, sensor was placed on bus no 3 and 8 .

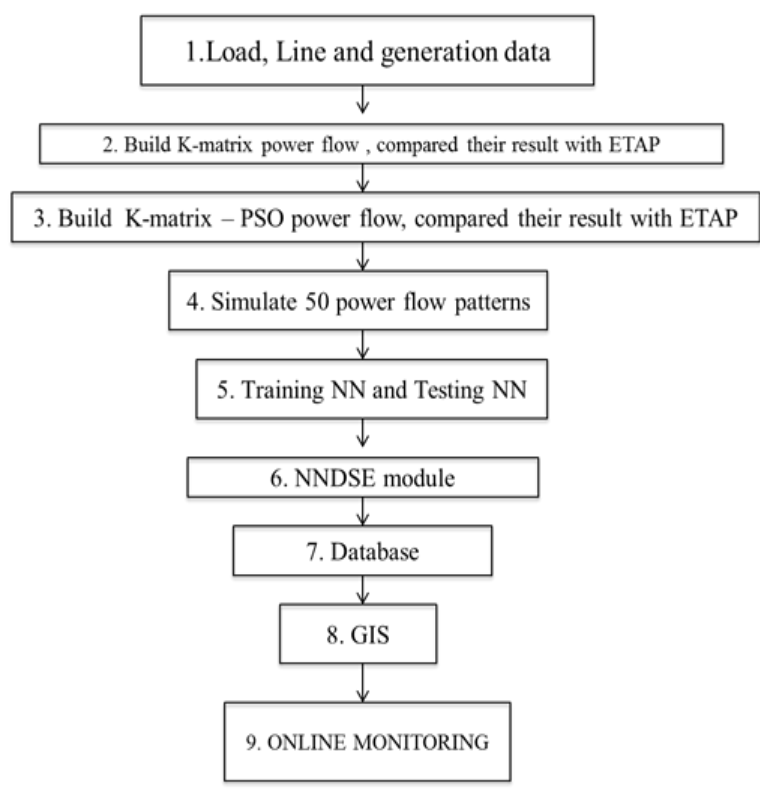

Fig. 2. Flow chart NN state estimation for active distribution systems.

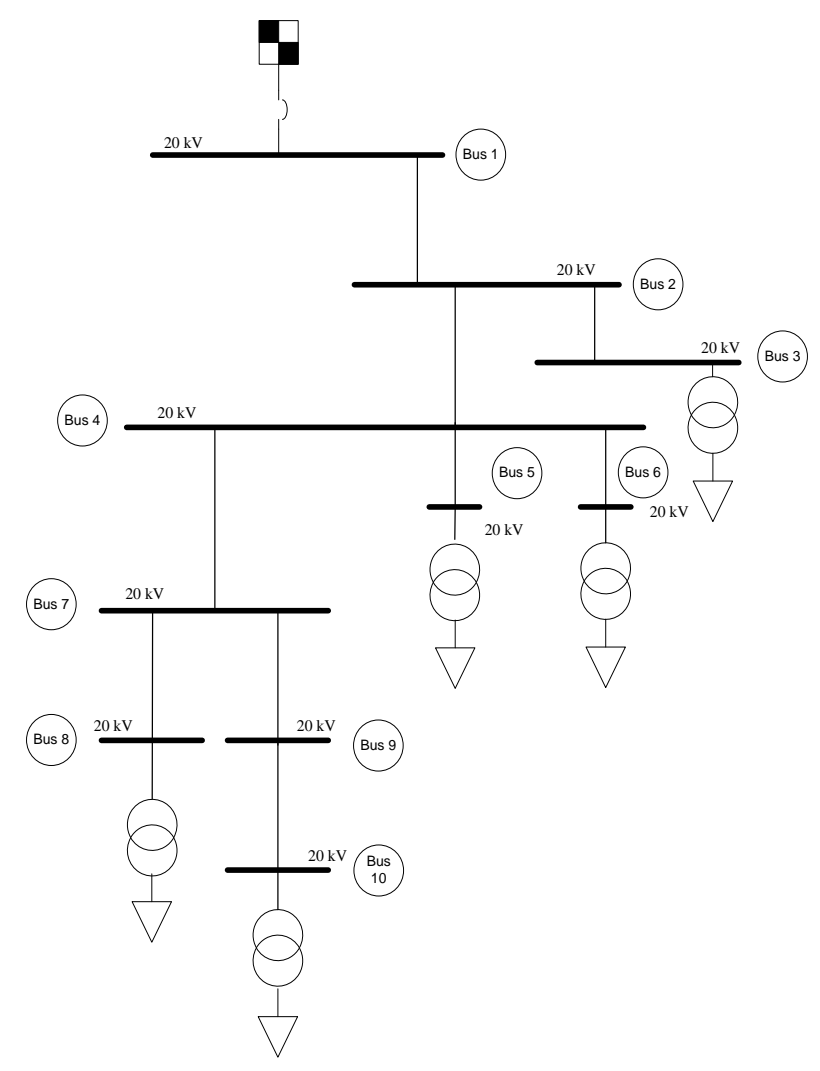

Fig. 3. Real distribution system, feeder of Kaliasin Surabaya Indonesia.

\section{Active Three Phase Distribution Network}

Case $\mathrm{C}$ is state estimation for three phase active distribution network. The data was used is feeder of Kaliasin. Same with Case B but it addition DG as PV bus at bus no3(see Table III).

Case 1 voltage estimation for bus no 5 and consider three input sensor from bus no 3, 6 and 10 .

Case 2 voltage estimation for bus no 5, 6 and 10, sensor was placed on bus no 3 and 8. Similar result between target and NNDSE are obtained. The proposed method accurately solves. The state estimation is displayed on GIS, it is shown in Fig. 4. Normal, under voltage and over voltage condition will 
be known. In Fig. 4 is an example that bus is under voltage. Fig. 5 showed the added menu in GIS to display the voltage magnitude and voltage angel for a node/bus in the systems.
For the next research will be combined between Neural Network and others Artificial Intelligent method to improve estimation result.

TABLE I: NN State Estimation In Single Phase Distribution NeTwORK

\begin{tabular}{|c|c|c|c|c|c|c|c|c|}
\hline & \multicolumn{4}{|c|}{ Case 1} & \multicolumn{4}{|c|}{ Case 2} \\
\hline & \multicolumn{2}{|c|}{ Voltage (pu) } & \multicolumn{2}{|r|}{ Angle } & \multicolumn{2}{|c|}{ Voltage (pu) } & \multicolumn{2}{|c|}{ Angle } \\
\hline \multirow{3}{*}{ Input } & $V_{2}$ & 0.99935 & $\theta_{2}$ & -0.0180 & $V_{2}$ & 0.99935 & $\theta_{2}$ & -0.0180 \\
\hline & $V_{5}$ & 0.99920 & $\theta_{5}$ & -0.0222 & \multirow{2}{*}{$V_{5}$} & \multirow{2}{*}{0.99916} & \multirow{2}{*}{$\theta_{5}$} & \multirow{2}{*}{-0.0233} \\
\hline & $V_{20}$ & 0.99906 & $\theta_{20}$ & -0.0253 & & & & \\
\hline \multirow{3}{*}{ Target } & $V_{5}$ & 0.99916 & $\theta_{5}$ & -0.0222 & $V_{5}$ & 0.99920 & $\theta_{5}$ & -0.0222 \\
\hline & \multirow{2}{*}{$V_{2}$} & \multirow{2}{*}{0.99916} & \multirow{2}{*}{$\theta_{5}$} & \multirow{2}{*}{-0.0233} & $V_{5}$ & 0.99920 & $\theta_{5}$ & -0.0222 \\
\hline & & & & & $V_{20}$ & 0.99096 & $\theta_{20}$ & -0.0253 \\
\hline \multirow{3}{*}{ NNDES } & $V_{5}$ & 0.99937 & $\theta_{5}$ & -0.0223 & $V_{5}$ & 0.99950 & $\theta_{5}$ & -0.0227 \\
\hline & \multirow{2}{*}{$V_{5}$} & \multirow{2}{*}{0.99927} & \multirow{2}{*}{$\theta_{5}$} & \multirow{2}{*}{-0.0238} & $V_{5}$ & 0.99970 & $\theta_{5}$ & -0.0229 \\
\hline & & & & & $V_{20}$ & 0.99916 & $\theta_{20}$ & -0.0260 \\
\hline \multirow{3}{*}{ Error } & $V_{5}$ & $0.017 \%$ & $\theta_{5}$ & $0.45 \%$ & $V_{5}$ & $0.03 \%$ & $\theta_{5}$ & $2.25 \%$ \\
\hline & \multirow{2}{*}{$V_{5}$} & \multirow{2}{*}{$0.011 \%$} & \multirow{2}{*}{$\theta_{5}$} & \multirow{2}{*}{$2.14 \%$} & $V_{5}$ & $0.05 \%$ & $\theta_{5}$ & $3.153 \%$ \\
\hline & & & & & $V_{20}$ & $0.872 \%$ & $\theta_{20}$ & $2.77 \%$ \\
\hline
\end{tabular}

TABLE II: NN State Estimation In PASsive Three Phase DistribUTION Network

\begin{tabular}{|c|c|c|c|c|c|c|c|c|c|c|c|c|c|}
\hline & & \multicolumn{4}{|c|}{ Phasa A } & \multicolumn{4}{|c|}{ Phasa B } & \multicolumn{4}{|c|}{ Phasa C } \\
\hline & & \multicolumn{2}{|c|}{ Voltage (pu) } & \multicolumn{2}{|c|}{ Angle } & \multicolumn{2}{|c|}{ Voltage (pu) } & \multicolumn{2}{|r|}{ Angle } & \multicolumn{2}{|c|}{ Voltage (pu) } & \multicolumn{2}{|c|}{ Angle } \\
\hline \multirow{9}{*}{ Case 1} & \multirow{3}{*}{ Input } & $V_{2}$ & 0.9995 & $\theta_{2}$ & -0.0180 & $V_{2}$ & 0.9993 & $\theta_{2}$ & -120.0197 & $V_{2}$ & 0.9992 & $\theta_{2}$ & 119.9821 \\
\hline & & $V_{5}$ & 0.9992 & $\theta_{5}$ & -0.0222 & $V_{5}$ & 0.9989 & $\theta_{5}$ & -120.0242 & $V_{5}$ & 0.9991 & $\theta_{5}$ & 119.9778 \\
\hline & & $V_{20}$ & 0.9990 & $\theta_{20}$ & -0.0253 & $V_{20}$ & 0.9990 & $\theta_{20}$ & -120.0276 & $V_{20}$ & 0.9989 & $\theta_{20}$ & 119.9748 \\
\hline & \multirow{2}{*}{ Target } & $V_{5}$ & 0.9994 & $\theta_{5}$ & -0.0222 & $V_{5}$ & 0.9992 & $\theta_{5}$ & -120.0242 & $V_{5}$ & 0.9915 & $\theta_{5}$ & 119.9779 \\
\hline & & $V_{5}$ & 0.9991 & $\theta_{5}$ & -0.0233 & $V_{5}$ & 0.9902 & $\theta_{5}$ & -120.0255 & $V_{5}$ & 0.9903 & $\theta_{5}$ & 119.9770 \\
\hline & \multirow{2}{*}{ NNDSE } & $V_{5}$ & 0.9993 & $\theta_{5}$ & -0.0225 & $V_{20}$ & 0.9994 & $\theta_{5}$ & -120.0280 & $V_{5}$ & 0.9925 & $\theta_{5}$ & 119.9400 \\
\hline & & $V_{5}$ & 0.9992 & $\theta_{5}$ & -0.0240 & $V_{5}$ & 0.9916 & $\theta_{5}$ & -120.0600 & $V_{5}$ & 0.9908 & $\theta_{5}$ & 119.9960 \\
\hline & \multirow{2}{*}{ Error } & $V_{5}$ & $0.01 \%$ & $\theta_{5}$ & $1.35 \%$ & $V_{5}$ & $0.02 \%$ & $\theta_{5}$ & $0.38 \%$ & $V_{5}$ & $0.1 \%$ & $\theta_{5}$ & $0.006 \%$ \\
\hline & & $V_{5}$ & $0.01 \%$ & $\theta_{5}$ & $3 \%$ & $V_{5}$ & $0.14 \%$ & $\theta_{5}$ & $0.028 \%$ & $V_{5}$ & $0.05 \%$ & $\theta_{5}$ & $0.015 \%$ \\
\hline \multirow{11}{*}{ Case 2} & \multirow{2}{*}{ Input } & $V_{2}$ & 0.9993 & $\theta_{2}$ & -0.0180 & $V_{2}$ & 0.9993 & $\theta_{2}$ & -120.0197 & $V_{2}$ & 0.9993 & $\theta_{2}$ & 119.9821 \\
\hline & & $V_{5}$ & 0.9991 & $\theta_{5}$ & -0.0233 & $V_{5}$ & 0.9991 & $\theta_{5}$ & -120.0255 & $V_{5}$ & 0.9991 & $\theta_{5}$ & 119.9960 \\
\hline & \multirow{3}{*}{ Target } & $V_{5}$ & 0.9992 & $\theta_{5}$ & -0.0222 & $V_{5}$ & 0.9992 & $\theta_{5}$ & -120.0280 & $V_{5}$ & 0.9992 & $\theta_{5}$ & 119.9400 \\
\hline & & $V_{5}$ & 0.9993 & $\theta_{5}$ & -0.0222 & $V_{5}$ & 0.9992 & $\theta_{5}$ & -120.0242 & $V_{5}$ & 0.9992 & $\theta_{5}$ & 119.9778 \\
\hline & & $V_{20}$ & 0.9909 & $\theta_{20}$ & -0.0253 & $V_{20}$ & 0.9909 & $\theta_{20}$ & -120.0276 & $V_{20}$ & 0.9909 & $\theta_{20}$ & 119.9749 \\
\hline & \multirow{3}{*}{ NNDSE } & $V_{5}$ & 0.9994 & $\theta_{5}$ & -0.0226 & $V_{5}$ & 0.9996 & $\theta_{5}$ & -120.0300 & $V_{5}$ & 0.9998 & $\theta_{5}$ & 119.9000 \\
\hline & & $V_{5}$ & 0.9995 & $\theta_{5}$ & -0.0226 & $V_{5}$ & 0.9994 & $\theta_{5}$ & -120.0200 & $V_{5}$ & 0.9993 & $\theta_{5}$ & 119.9600 \\
\hline & & $V_{20}$ & 0.9910 & $\theta_{20}$ & -0.0251 & $V_{20}$ & 0.9923 & $\theta_{20}$ & -120.0400 & $V_{20}$ & 0.9920 & $\theta_{20}$ & 119.9800 \\
\hline & \multirow{3}{*}{ Error } & $V_{5}$ & $0.02 \%$ & $\theta_{5}$ & $1.8 \%$ & $V_{5}$ & $0.04 \%$ & $\theta_{5}$ & $0.001 \%$ & $V_{5}$ & $0.06 \%$ & $\theta_{5}$ & $0.03 \%$ \\
\hline & & $V_{5}$ & $0.03 \%$ & $\theta_{5}$ & $1.8 \%$ & $V_{5}$ & $0.02 \%$ & $\theta_{5}$ & $0.003 \%$ & $V_{5}$ & $0.01 \%$ & $\theta_{5}$ & $0.01 \%$ \\
\hline & & $V_{20}$ & $0.01 \%$ & $\theta_{20}$ & $0.88 \%$ & $V_{20}$ & $0.14 \%$ & $\theta_{20}$ & $0.01 \%$ & $V_{20}$ & $0.11 \%$ & $\theta_{20}$ & $0.004 \%$ \\
\hline
\end{tabular}


TABLE III: NN State Estimation IN ACtive Three Phase Distribution NeTwork

\begin{tabular}{|c|c|c|c|c|c|c|c|c|c|c|c|c|c|}
\hline & & \multicolumn{4}{|c|}{ Phasa A } & \multicolumn{4}{|c|}{ Phasa B } & \multicolumn{4}{|c|}{ Phasa C } \\
\hline & & \multicolumn{2}{|c|}{ Voltage (pu) } & \multicolumn{2}{|c|}{ Angle } & \multicolumn{2}{|c|}{ Voltage (pu) } & \multicolumn{2}{|r|}{ Angle } & \multicolumn{2}{|c|}{ Voltage (pu) } & \multicolumn{2}{|r|}{ Angle } \\
\hline \multirow{9}{*}{ Case 1} & \multirow{3}{*}{ Input } & $V_{3}$ & 1 & $\theta_{3}$ & -0.0721 & $V_{3}$ & 1 & $\theta_{3}$ & -120.0781 & $V_{3}$ & 1 & $\theta_{3}$ & 119.9237 \\
\hline & & $V_{6}$ & 0.9997 & $\theta_{6}$ & -0.0752 & $V_{6}$ & 0.9997 & $\theta_{6}$ & -120.0815 & $V_{6}$ & 0.9996 & $\theta_{6}$ & 119.9205 \\
\hline & & $V_{10}$ & 0.9994 & $\theta_{10}$ & -0.0783 & $V_{10}$ & 0.9994 & $\theta_{10}$ & -120.0848 & $V_{10}$ & 0.9994 & $\theta_{10}$ & 119.9176 \\
\hline & \multirow{2}{*}{ Target } & $V_{5}$ & 0.9997 & $\theta_{5}$ & -0.0752 & $V_{5}$ & 0.9997 & $\theta_{5}$ & -120.0814 & $V_{5}$ & 0.9996 & $\theta_{5}$ & 119.9206 \\
\hline & & $V_{8}$ & 0.9996 & $\theta_{8}$ & -0.0763 & $V_{8}$ & 0.9996 & $\theta_{8}$ & -120.0828 & $V_{8}$ & 0.9995 & $\theta_{8}$ & 119.9197 \\
\hline & \multirow{2}{*}{ NNDSE } & $V_{5}$ & 0.9997 & $\theta_{5}$ & -0.0760 & $V_{5}$ & 0.9997 & $\theta_{5}$ & -120.0700 & $V_{5}$ & 0.9995 & $\theta_{5}$ & 119.3000 \\
\hline & & $V_{8}$ & 0.9997 & $\theta_{8}$ & -0.0770 & $V_{8}$ & 0.9998 & $\theta_{8}$ & -120.7500 & $V_{8}$ & 0.9954 & $\theta_{8}$ & 119.9400 \\
\hline & \multirow{2}{*}{ Error } & $V_{5}$ & $0.02 \%$ & $\theta_{5}$ & $1.06 \%$ & $V_{5}$ & $0.02 \%$ & $\theta_{5}$ & $0.0095 \%$ & $V_{5}$ & $0.01 \%$ & $\theta_{5}$ & $0.52 \%$ \\
\hline & & $V_{8}$ & $0.02 \%$ & $\theta_{8}$ & $0.917 \%$ & $V_{8}$ & $0.01 \%$ & $\theta_{8}$ & $0.55 \%$ & $V_{8}$ & $0.4 \%$ & $\theta_{8}$ & $0.017 \%$ \\
\hline \multirow{11}{*}{ Case 2} & \multirow{2}{*}{ Input } & $V_{3}$ & 1 & $\theta_{3}$ & -0.0721 & $V_{3}$ & 1 & $\theta_{3}$ & -120.0781 & $V_{3}$ & 1 & $\theta_{3}$ & 119.9237 \\
\hline & & $V_{8}$ & 0.9991 & $\theta_{8}$ & -0.0763 & $V_{8}$ & 0.9991 & $\theta_{8}$ & -120.0828 & $V_{8}$ & 0.9996 & $\theta_{8}$ & 119.9197 \\
\hline & \multirow{3}{*}{ Target } & $V_{5}$ & 0.9997 & $\theta_{5}$ & -0.0752 & $V_{5}$ & 0.9997 & $\theta_{5}$ & -120.0814 & $V_{5}$ & 0.9996 & $\theta_{5}$ & 119.9206 \\
\hline & & $V_{6}$ & 0.9997 & $\theta_{6}$ & -0.0752 & $V_{6}$ & 0.9997 & $\theta_{6}$ & -120.0815 & $V_{6}$ & 0.9996 & $\theta_{6}$ & 119.9205 \\
\hline & & $V_{10}$ & 0.9994 & $\theta_{10}$ & -0.0763 & $V_{10}$ & 0.9994 & $\theta_{10}$ & -120.0848 & $V_{10}$ & 0.9994 & $\theta_{10}$ & 119.9176 \\
\hline & \multirow{3}{*}{ NNDSE } & $V_{5}$ & 0.9997 & $\theta_{5}$ & -0.0752 & $V_{5}$ & 0.9997 & $\theta_{5}$ & -120.0814 & $V_{5}$ & 0.9996 & $\theta_{5}$ & 119.9206 \\
\hline & & $V_{6}$ & 0.9997 & $\theta_{6}$ & -0.0752 & $V_{6}$ & 0.9997 & $\theta_{6}$ & -120.0815 & $V_{6}$ & 0.9996 & $\theta_{6}$ & 119.9205 \\
\hline & & $V_{10}$ & 0.9997 & $\theta_{10}$ & -0.0752 & $V_{10}$ & 0.9994 & $\theta_{10}$ & -120.0848 & $V_{10}$ & 0.9996 & $\theta_{10}$ & 119.9176 \\
\hline & \multirow{3}{*}{ Error } & $V_{5}$ & $0.01 \%$ & $\theta_{5}$ & $0.27 \%$ & $V_{5}$ & $0.03 \%$ & $\theta_{5}$ & $0.008 \%$ & $V_{5}$ & $0.01 \%$ & $\theta_{5}$ & $0.008 \%$ \\
\hline & & $V_{6}$ & $0.01 \%$ & $\theta_{6}$ & $0.93 \%$ & $V_{6}$ & $0.01 \%$ & $\theta_{6}$ & $0.0079 \%$ & $V_{6}$ & $0.01 \%$ & $\theta_{6}$ & $0.0004 \%$ \\
\hline & & $V_{10}$ & $0.01 \%$ & $\theta_{10}$ & $0.13 \%$ & $V_{10}$ & $0.01 \%$ & $\theta_{10}$ & $0.012 \%$ & $V_{10}$ & $0.02 \%$ & $\theta_{10}$ & $0.019 \%$ \\
\hline
\end{tabular}

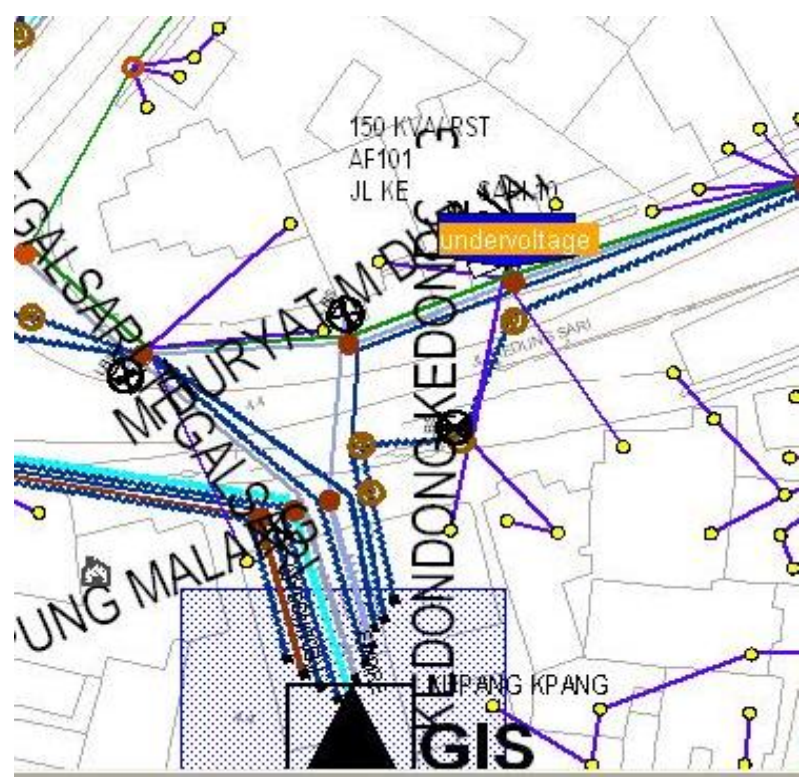

Fig. 4. State estimation displayed in GIS.

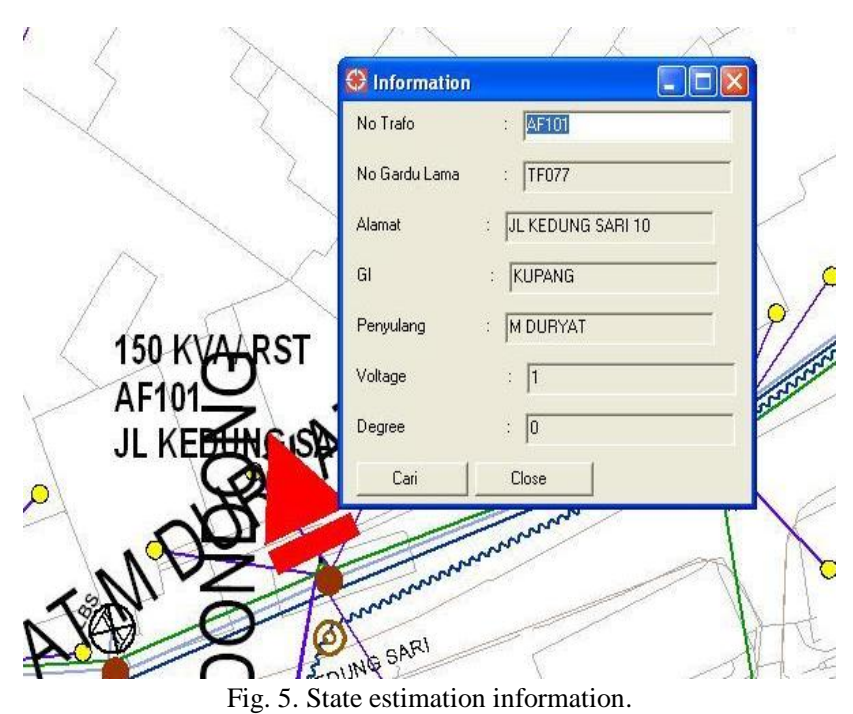

Fig. 5. State estimation information.

\section{CONCLUSION}

The proposed method which is a new approach to solve for active unbalanced distribution networks accurately. The simulation results show that proposed method can accommodate PV bus. State estimation using neural network for all case have error under $4 \%$ and reduce sensor almost $50 \%$.

\section{ACKNOWLEDGMENT}

The authors gratefully acknowledge the support by. Indonesian Government, East-Java Electricity Distribution Company (PLN), Realworld Indonesia.

\section{REFERENCES}

[1] F. C. Schweppe and J. Wildes, "Power system static-state estimation, part I: exact model," IEEE Trans. Power App. Syst., vol. PAS-89, pp.120-125, Jan. 1970.

[2] F. C. Schweppe and D. B. Rom, "Power system static-state estimation, part II: approximate model," IEEE Trans. Power App. Syst., vol. PAS-89, pp. 125-130, Jan. 1970.

[3] F. C. Schweppe, "Power system static-state estimation, part III: implementation," IEEE Trans. Power App. Syst., vol. PAS-89, pp. 262-280, Jan. 1970.

[4] J. Wan and K. N. Miu, "Weighted least squares methods for load estimationin distribution networks," IEEE Trans. Power Syst., vol. 18, no. 4, pp. 1338-1345, Nov. 2003.

[5] K. Li, "State estimation for power distribution system and measurementimpacts," IEEE Trans. Power Syst., vol. 11, no. 2, pp. 911-916, May 1996.

[6] M. Almeida, D. Silveira, and M. F. de Medeiros, Jr., "Estimating loads in distribution feeders using a state estimator algorithm with additionaladjustment of transformers loading factors," in Proc. IEEE Int. Symp.Circuits Syst., Bangkok, Thailand, Mar. 2003, vol. 5, pp. III 328-III 321.

[7] J. H. Teng, "A network-topology based three: phase load flow for distribution systems," National Science Council ROC (A), vol. 24, no. 4, pp. 259-264, 2000.

[8] T. H. Chen and N. C. Yang, Three-phase power-flow by direct $Z_{B R}$ method for unbalanced radial distribution systems. Generation, Transmission \& Distribution, IET, vol. 3, no. 10, pp. 903-910, 2009.

[9] Mat Syai'in, K. L. L., N. C. Yang, and T. H. Chen, “A distribution power flow using particle swarm optimization," presented at the IEEE PES General Meeting, 22 - 26 July 2012, San Diego, CA, USA. 
[10] M. Syai'in and O. Penangsang., "Adi suprijanto, real-time unbalanced load flow development using direct-ZBR method and modified lambda iteration for on-line monitoring and control," presented at CIRED, Stockholm, Sweden, July 2013

[11] M. Syai'in, O. Penangsang., and A. Suprijanto, "Voltage estimation using Direct $Z_{\mathrm{BR}}+$ Modified lambda iteration for on-line monitoring in electrical power distribution system," presented at IEEE, 2012, Kyoto.

[12] T. H. Chen and N. C. Yang, "Three-phase power-flow by direct ZBR method for unbalanced radial distribution systems," Generation, Transmission \& Distribution, IET, vol. 3, no. 10, pp. 903-910, 2009.

[13] P. A. N. Garcia et al., "Three-phase power flow calculations using the current injection method," IEEE Transactions on Power Systems, vol. 15 , no. $2,2000$.

[14] V. M. da Costa et al.,"Developments in Newton Raphson power flow formulation based on current injections," IEEE Transactions on Power System. vol. 14, no. 4, 1999.

[15] W. H. Kersting, Distribution System Modeling and Analysis, CRC Press, 2000.

[16] J. H. Teng, "A network-topology based three: phase load flow for distribution systems," National Science Council ROC (A), vol. 24, no. 4, pp. pp. 259-264, 2000.

[17] K. L. Lo and C. Zhang, "Decomposed three-phase power flow solution using the sequence component frame," Generation, Transmission and Distribution, IEE Proceedings C. vol. 140, no. 3, pp. 181-188, 1993.

[18] M. Abdel-Akher, K. M. Nor, and A. H. A. Rashid, "Improved Three-Phase Power-Flow Methods Using Sequence Components," IEEE Transactions on Power Systems, vol. 20, no. 3, pp. 1389-1397, 2005.

[19] M. Z. Kamh and R. Iravani, "Unbalanced Model and Power-Flow Analysis of Microgrids and Active Distribution Systems," IEEE Transactions on Power Delivery, vol. 25, no. 4, pp. 2851- 2858, 2010.

[20] M. Z. Kamh, "A unified three phase power flow analysis model for electronically coupled distributed energy resources," IEEE Trans. Power Deliv., vol. 26, no. 2, pp. 899-909, 2011.

[21] J. Kenedy, "Particle swarm optimization," IEEE Trans., vol. 26, no. 2, pp. 1942-1048, 1995.

[22] M. Biserica et al., "Neural networks to improve distribution state estimation - volt var control performances," IEEE Trans. SMART GRID.,vol. 3, no. 3, pp. 1137-1144, 2012.

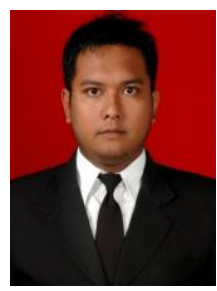

Dimas Fajar Uman P. was born in Surabaya on November 8, 1988. He finished his undergraduates program from Electrical Engineering Department ITS Surabaya in 2010 and then he continued his study for master program in Electrical Engineering Department ITS Surabaya and finished it in 2012, now he studying at doctoral program in Electrical Engineering Department ITS Surabaya.

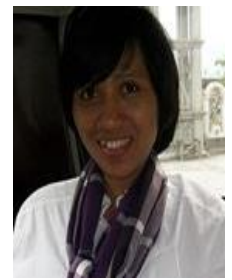

Indri Suryawati was born in Tuban on October 1, 1983. She received her diploma from Electronics Engineering Polytechnic Institute of Surabaya in 2004. Finished her undergraduates program in Electrical Engineering Department of ITS Surabaya in 2010. Now she is study at master program in Electrical Engineering Department of ITS Surabaya.

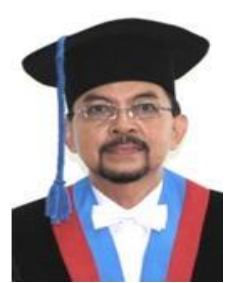

Ontoseno Penangsang was born in Indonesia. $\mathrm{He}$ received the B.E. degree in electrical engineering from Institut Teknologi Sepuluh Nopember, Surabaya, Indonesia, in 1974 and M.S degree in electrical engineering from University of Wisconsin-Madison College of Engineering, USA, in 1978. He received the Ph.D degree in electrical engineering from University of Wisconsin-Madison College of Engineering, USA, in 1983. Since 1974, he has been a lecturer in the Department of the Electrical Engineering, Institut Teknologi Sepuluh Nopember, and Surabaya, Indonesia. His current research interests include the application computational intelligence application on power system, power system operation and control, power system analysis, power system stability and power quality.

$\mathrm{He}$ is currently a professor of Department of Electrical Engineering, Sepuluh November Institute of Technology (ITS).

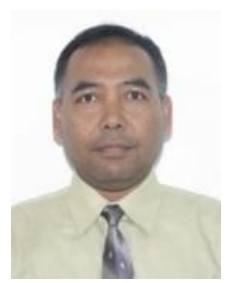

Adi Soeprijanto was born in Indonesia. He received his B.E. and M.S. degrees in electrical engineering from Institut Teknologi Bandung, Bandung, Indonesia, in 1988 and 1995, respectively. He received the $\mathrm{Ph} . \mathrm{D}$. degree in electrical engineering from Hiroshima University in 2001. Since 1990, he has been a lecturer in the Department of the Electrical Engineering, Institut of Teknologi Sepuluh Nopember, in Surabaya, Indonesia. His current research interests include the application of intelligent systems to power system operation, management, and control.

Prof. Dr. Ir. Adi Soeprijanto is a member of the Indonesian Power System Expert Association.

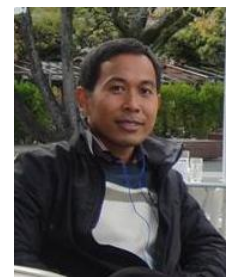

Matt Syai'in was born in Indonesia

B.E. degree in engineering physics, M.S degree and doctoral in electrical engineering from Sepuluh Nopember Institute of Technology, Surabaya, Indonesia, in 2003, 2008 and 2012, respectively.

Since 2008, he has been a lecturer in the Shipbuilding State Polytechnics, Sepuluh Nopember Institute of Technology, Surabaya, Indonesia 